\title{
Correlation between the magnetic properties and the crystallographic texture during the processing of non oriented electrical steel
}

\author{
Kim Verbeken ${ }^{1, a}$, Edgar Gomes ${ }^{1, b}$, Jürgen Schneider ${ }^{1, c}$ and Yvan Houbaert ${ }^{1, d}$ \\ ${ }^{1}$ Ghent University (UGent), Department of Materials Science and Engineering, \\ Technologiepark 903, B-9052 Gent (Zwijnaarde), Belgium \\ akim.verbeken@ugent.be, ${ }^{b} E d g a r . G o m e s @ U G e n t . b e,{ }^{c}$ schneider.juergen@t-online.de, \\ 'Yvan.Houbaert@UGent.be
}

\begin{abstract}
Keywords: texture, non oriented electrical steel, magnetic properties, thermo-mechanical processing
\end{abstract}

\begin{abstract}
The magnetic properties in electrical steels are strongly dependent on the crystallographic texture as well as other microstructural features such as grain size. Both, texture and grain size, are determined by the thermo-mechanical history of the material. This work regards a set of different thermo-mechanical paths applied on two types of non-oriented electrical steels containing $2.4 \%$ and $3.0 \% \mathrm{Si}$, respectively. The evolution of grain size, microstructure morphology and texture throughout processing were studied in detail by optical microscopy, X-Ray diffraction and Electron BackScatter Diffraction (EBSD). The impact of the texture on the magnetic properties was evaluated. This was done by the calculation of the magnetic anisotropy energy and the A parameter, i.e. a parameter defined in scientific literature that describes the magnetic "quality" of the texture, which can be correlated with the magnetic properties of the materials. Finally, the influence of further laser cutting on the crystallographic texture will be examined as well.
\end{abstract}

\section{Introduction}

The magnetization behaviour is one of the most frequently used examples to illustrate the anisotropy of the bcc crystal lattice of iron, since it is well known that $\langle 100\rangle$ directions are much more easily magnetized than $\langle 110\rangle$ and $\langle 111\rangle$ directions. Consequently, the magnetic properties of electrical steels, i.e. the magnetization behaviour and the specific magnetic losses, are strongly influenced by the microstructural and textural characteristics of the material. Apart from the crystallographic orientations and grain size, also inclusions, internal stresses and surface defects are reported to determine the domain wall pinning, i.e. the coercive force of the material and the domain wall motion, which are responsible for the hysteresis losses and the magnetizing behaviour at low and medium values of the applied external magnetic field. In polycrystalline materials, the crystallographic texture determines the remanent magnetic induction and the domain rotation processes at high values of the applied external magnetic field.

For steels, the steel type determines the desired texture and microstructure, which are aimed for after thermo-mechanical processing. The processing parameters, such as the amount of deformation during hot and cold rolling or the annealing time and temperature during the final annealing treatment, are employed to steer the texture into the wanted direction. In more conventional steels, such as deep drawing qualities, a high intensity on the gamma $(\{111\}\langle u v w\rangle)$ fibre and a small grain size is wanted in order to improve the formability, and a lot of research has been published on this topic, e.g. [1]. On the other hand, in electrical steels, large grains are beneficial because they lower the hysteresis losses and the gamma fibre orientations are unwanted because of their inferior magnetization behaviour. In order to obtain low magnetic losses and high permeability, the preferable texture component for grain oriented electrical steels is the Goss $(\{110\}<001>)$ texture, while an intense distribution of orientations along the theta $(\{100\}\langle\mathrm{uvw}\rangle)$ fibre is wanted for nonoriented steels. 
Both types of electrical steel have already been studied extensively in scientific literature. With respect to the grain oriented steels, a lot of research has been published on the development of the Goss texture component, e.g. [2-4]. For non-oriented steels, many researchers have studied the effect of varying the thermo-mechanical processing parameters in order to obtain a magnetically more favourable crystallographic texture. It has been shown that a hot band annealing improves the magnetic properties [5-6]. This annealing, especially for Fe-Si alloys with phase transformation, leads to an enhanced intensity of the Goss and cube texture as well as of the eta $(\{\mathrm{hkl}\}<100>)$ fibre. Similar effects may be obtained by choosing higher coiling temperatures after hot rolling [7]. It has also been demonstrated that a coarse grained hot band structure gives a higher intensity on the Goss texture component [8-9]. A final hot rolling step in the two phase region and in the ferritic region may also result in a better magnetization behaviour of the materials [10-11]. Apart from sticking to a more conventional processing route some suggestions have been made to leave the conventional route in order to obtain a more significant improvement of the final texture. One example that gave an exceptional improvement of the final texture was the application of cross rolling [12]. In this process, the rolling direction during hot rolling became the transverse direction during cold rolling. When cold rolling and annealing was followed by additional rolling, with a small reduction, and annealing treatment, a very high intensity, nicely distributed along the desired theta fibre was obtained. However, the industrial applicability of this process is not self-evident.

Whereas a lot of the work on non-oriented electrical steels concentrates on steels with a Si content that is low enough to still have an austenite-ferrite phase transformation, in the current work, the Si content will be high enough to suppress this phase transformation. Some results will be shown from an extensive variation of the hot rolling parameters. The magnetic quality of the obtained textures will be discussed after defining an appropriate parameter to describe this feature. Finally, some indications of the impact of further cutting processes will be given as well.

\section{Experimental procedure}

Before hot rolling, the materials were annealed at $1260{ }^{\circ} \mathrm{C}$. Hot rolled samples of Fe-2.4wt $\%$ Si and Fe-3.0wt $\%$ Si steel with $80 \mathrm{~mm}$ width and $2 \mathrm{~mm}$ thickness were fabricated using the four stand high speed hot rolling mill at TU Freiberg. The final thickness was reached after six passes. The reduction was larger than $40 \%$ in the first five passes. The finishing temperature (FT) and the subsequent cooling conditions to $200{ }^{\circ} \mathrm{C}$ were varied over a wide range of parameters. On the one hand, FT varied between $700{ }^{\circ} \mathrm{C}$ and $1100{ }^{\circ} \mathrm{C}$ and on the other hand, after finishing, the samples were rapidly cooled to temperatures ranging from $400{ }^{\circ} \mathrm{C}$ to $950{ }^{\circ} \mathrm{C}$ and followed by a variable holding time and temperature. Finally, the samples were slowly cooled to $200{ }^{\circ} \mathrm{C}$ at a constant rate of $50 \mathrm{~K} / \mathrm{h}$. After hot rolling, the samples were cold rolled to a thickness of $0.50 \mathrm{~mm}$. Finally, annealing was realized in a laboratory furnace to simulate the continuous annealing process. The samples were rapidly heated to about $950{ }^{\circ} \mathrm{C}$ and kept at this temperature for $45 \mathrm{~s}$ and finally cooled to $200{ }^{\circ} \mathrm{C}$ in $300 \mathrm{~s}$.

Besides metallographic investigations with optical microscopy, the Electron BackScatter Diffraction (EBSD) technique on a FEI XL30 ESEM equipped with a LaB 6 -filament and XRD on a Siemens D5000 diffractometer, using $\operatorname{MoK}_{\alpha}$ radiation $(\lambda=0.070926 \mathrm{~nm})$ were combined for detailed crystallographic analysis of the samples. Step sizes for EBSD were 0.8, 5 and $10 \mu \mathrm{m}$. The homogeneity of the grain size and texture across the thickness was also analyzed.

Besides the laboratory materials, commercially produced non-oriented Fe-Si steels with thickness of $0.5 \mathrm{~mm}$ and medium silicon content were used to study the effect of further cutting. The microstructure was studied by optical metallography and EBSD.

\section{Evolution of microstructure and texture during thermo-mechanical processing}

From the vast amount of laboratory material, four samples were chosen. Their hot rolling parameters are summarized in Table 1. The evolution of the texture and microstructure during 
thermo-mechanical processing was discussed in detail elsewhere [13, 14] and are briefly summarized here.

\begin{tabular}{cccccc} 
Sample Name & Si Content & Finishing Temperature & Rapid Cooling To & $\begin{array}{c}\text { Holding Temperature } \\
\text { (time) }\end{array}$ & Slow Cooling To \\
\hline I & $2.4 \%$ & $860^{\circ} \mathrm{C}$ & $800^{\circ} \mathrm{C}$ & $750^{\circ} \mathrm{C}(20 \mathrm{~min})$ & $200^{\circ} \mathrm{C}$ \\
II & $2.4 \%$ & $820^{\circ} \mathrm{C}$ & $400^{\circ} \mathrm{C}$ & - & $200^{\circ} \mathrm{C}$ \\
III & $3.0 \%$ & $860^{\circ} \mathrm{C}$ & $800^{\circ} \mathrm{C}$ & $750^{\circ} \mathrm{C}(2 \mathrm{~min})$ & $200^{\circ} \mathrm{C}$ \\
IV & $3.0 \%$ & $820^{\circ} \mathrm{C}$ & $400^{\circ} \mathrm{C}$ & - & $200^{\circ} \mathrm{C}$ \\
\hline
\end{tabular}

Table 1: Summary of the hot rolling parameters for the different samples studied.

It was found that rapid cooling at finishing gave rise to a deformed microstructure across the thickness. An interrupted cooling including holding of the sample at $750{ }^{\circ} \mathrm{C}$ caused recovery and recrystallization near the sample surface, but the central region still remained deformed. The texture at the surface was mainly dominated by the $\{110\}<001>$ and $\{112\}<111>$ shear texture components while in the deformed region, the highest intensity was found at the rotated cube $\{001\}<110\rangle$ component. Steel III differed from the other steels and was found to have a quite weak texture with small intensity maxima at the rotated cube component and near the $\{111\}<112\rangle$ component on the gamma fibre.

After cold rolling, both alpha $(\{\mathrm{hkl}\}<110>)$ and gamma $(\{111\}<\mathrm{uvw}>)$ fibre components were found. Generally, a higher intensity was found at the rotated cube component when the material had a more pronounced rotated cube component after hot rolling. After annealing, as is illustrated in Figure 1, the highest texture intensities were found at the $\{111\}<112>$ component on the gamma fibre. Also components along a secondary fibre, parallel to the alpha fibre, running from the $\{111\}<112>$ component to the theta fibre were identified. This fibre was already reported in literature for IF [15] and a ULC [16] steel. These results showed that despite significant differences in the intensity distributions of the hot rolling textures, further conventional thermo-mechanical processing resulted in quite similar textures after full processing.
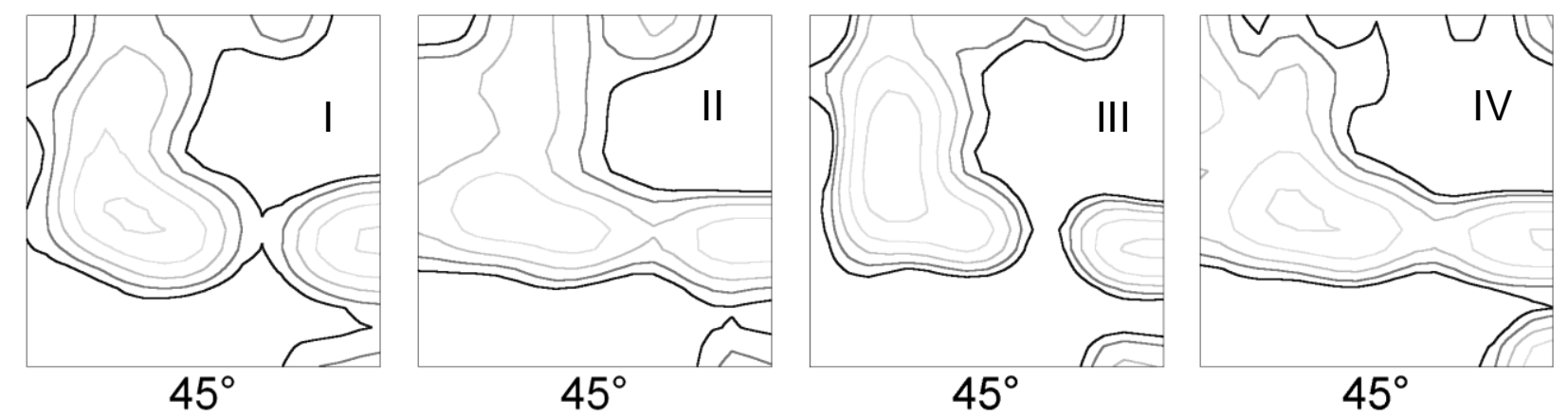

Fig. 1: $\varphi_{2}=45^{\circ}$ section of ODF of the materials given in Table 1 after cold rolling and annealing. Levels: 1.0 - $1.4-2.0-2.8-4.0-5.7$.

\section{Description of the magnetic quality of the texture}

In order to describe and quantify the magnetic texture quality of a non-oriented electrical steel, two alternatives are available. A first one was defined by Kestens and Van Houtte [17] and can be derived from the experimentally determined orientation distribution function (ODF), which is reflected in the function $\mathrm{f}(\mathrm{g})$. The orientation $\mathrm{g}$ is defined by its Euler angles. A schematic illus tration is given in Fig. 2. For each ODF data point, the angle is determined which is the minimum angle of the three angles $\alpha_{1}, \alpha_{2}$ and $\alpha_{3}$ between one of the $\langle 100\rangle$ directions of easy magnetization of the crystal in the polycrystalline material and the direction of the macroscopic magnetization vector $M$ in the material. The externally applied magnetic field, which is represented by this magnetization vector $M$, makes an angle $\theta$ with respect to the rolling direction (RD) of the sheet. 
For the sake of simplicity $\theta$ is taken 0 in Fig. 2. The volume fraction of orientations in an infinitesimal environment of $\mathrm{g}$ is given by $\mathrm{f}(\mathrm{g}) \mathrm{dg}$. Finally, a texture parameter $\mathrm{A}_{\theta}$ is defined in Eq. (1).

$$
A_{\vartheta}=\int f(g) A(g) d g
$$

Where $A_{\theta}$ is the texture parameter in the case that the magnetic field vector $M$ is applied in a direction making an angle $\theta$ with the rolling direction. The value of $\mathrm{A}_{\theta}$ ranges from 0 to $54.7^{\circ}$. In a non-oriented electrical steel that is typically used in a rotating part of an electrical machine, the direction of $\mathrm{M}$ rotates with respect to the rolling direction of the material and an average $\mathrm{A}$ parameter is defined as determined by Eq. 2 .

$$
A=\int A_{\vartheta} d \theta
$$

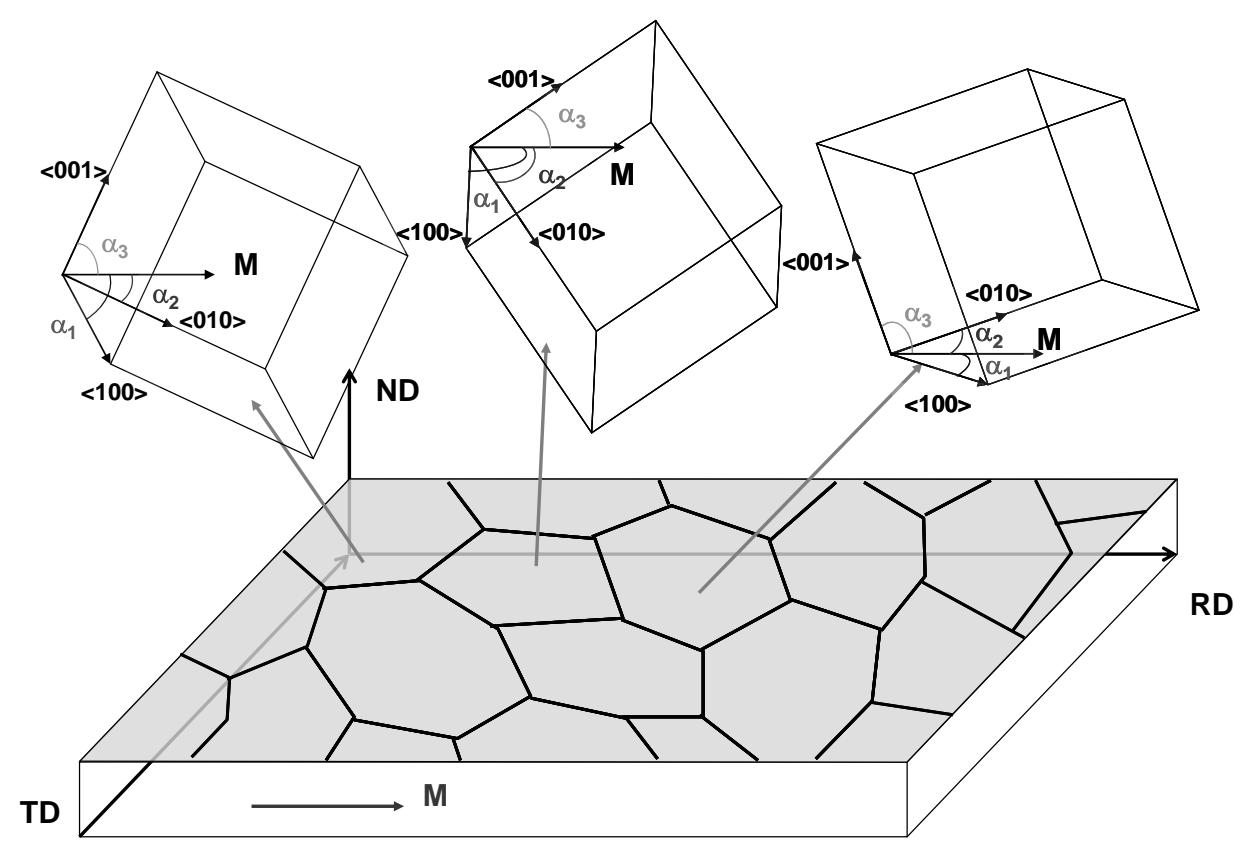

Fig. 2: Schematic illustration on how the minimum angle between the magnetization vector $M$ and closest easy magnetization $<100\rangle$ direction is defined.

An alternative way to describe the magnetic quality of a non-oriented electrical steel might be the magnetic anisotropy energy $\mathrm{E}_{\mathrm{a}}$. In order to study whether both descriptions are equivalent, Gomes et al. [18] calculated both $\mathrm{E}_{\mathrm{a}}$ and the A parameter for several $\mathrm{Fe}-\mathrm{Si}$ steels with variable $\mathrm{Si}$ content. A perfectly linear relationship was found between both parameters, which demonstrated that both parameters can be used to describe the magnetic quality of the steel. Moreover, it was also shown elsewhere [18] that the A-parameter, together with the grain size and a parameter related with the chemical composition of the steel could nicely predict the magnetic induction $\mathrm{B}$ at different values of the applied field $\mathrm{H}$, ranging from 500 to $5000 \mathrm{~A} / \mathrm{m}$. Therefore, the present work will use the Aparameter as a measure for the magnetic quality of the material.

A graph showing the evolution of the A-parameter as a function of the angle $\theta$ for some ideal texture components and fibres is given in [19]. It was shown there that a perfect theta fibre has an averaged A-parameter of 22.5, which is the same value as for the cube and rotated cube component. The gamma fibre, on the other hand, has an A-parameter of 38.7, the value of the alpha fibre is 30.1, whereas a perfectly random texture would correspond with a value of 31.9.

When looking at the evolution of the A-parameter, it was found that after hot rolling the parameter was significantly higher, i.e. worse, for steel III as could be expected when looking at the features of the texture of this steel. After cold rolling, this difference in A-parameter diminished and it almost completely disappeared after subsequent annealing, as could be expected from Fig. 1. Despite the wide variation in the parameters during the hot rolling process and the significant 
differences in the A-parameters obtained after hot rolling, it could be concluded that further cold rolling and annealing weakened these variations resulting in materials that were with respect to their magnetic quality not optimal.

\section{Effect of laser cutting on the magnetic properties}

Apart from aiming at obtaining a magnetically favourable texture after thermo-mechanical processing, further cutting processes can also significantly alter the microstructure and texture near the cut edge. Whereas in the case of mechanical cutting there is a clear region with changes in the grain morphology near the cut edge due to the plastic deformation there is no clear indication of a change of the grain morphology for samples obtained by laser cutting, as can be seen in Fig. 3(a). It has, however, been demonstrated [20] that laser cutting causes a deterioration of the magnetic properties. It was argued that residual biaxial stresses due to the thermal shock wave at laser cutting may be the origin of the observed changes of the remanent induction and the decrease of the permeability at higher magnetic fields, respectively.

As can be seen in Fig. 3, the texture is significantly altered by the laser cutting process. Fig. 3(b) illustrates the difference in texture between an area near the cut edge for a sample prepared by laser cutting and the texture in the sample interior by making use of the $\varphi_{2}=45^{\circ}$ section of the ODF. It was observed that in the area near the cut edge mainly orientations appeared that were away from the gamma fibre. Other work [21] identified similar texture components along the $\{\mathrm{h} 11\}<1 / \mathrm{h}, 1,2>$ fibre after severe rolling (total strain exceeding 4.6) and annealing of a Fe-3wt\%Si steel. The ODF intensity lines of region A are mainly concentrated along this fibre, although it must also be mentioned that the statistics are rather poor, because of the limited number of grains in the area affected by laser cutting. The reported texture changes near the cut line could be correlated with the thermal energy put into the material during laser cutting, which is also believed to be responsible for the deterioration of the magnetic properties.
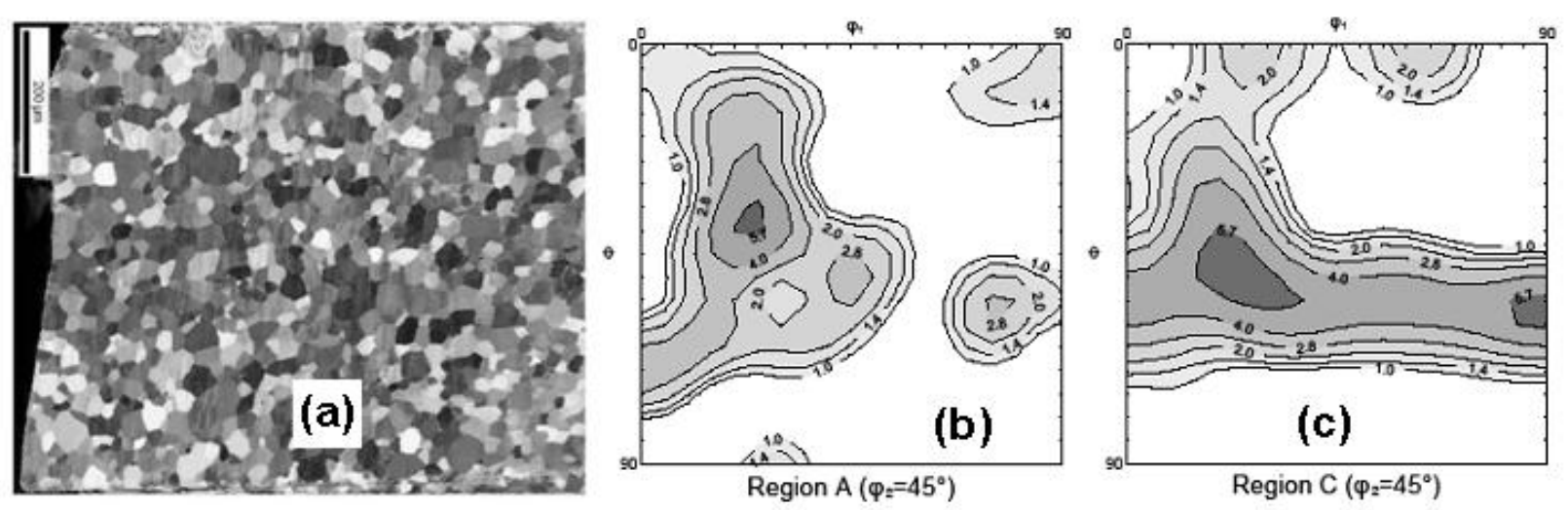

Fig. 3: (a) Inverse pole figure map of a non oriented Fe-Si steel with medium Si-content after laser cutting, (b) $\varphi_{2}=45^{\circ}$ section of the ODF obtained by EBSD of the same steel within a distance of $50 \mu \mathrm{m}$ from the cut edge (c) $\varphi_{2}=45^{\circ}$ section of the ODF obtained by EBSD in a region unaffected by cutting, levels: $1.0-1.4-2.0-2.8-4.0-5.7$.

\section{Conclusions}

In two non oriented electrical steels, with a Si content that was high enough to avoid phase transformations, extensive variations in the hot rolling parameters were imposed as a possible way to improve the final texture. Both texture evaluation and A-parameter calculation indicated that the initial improvements of the texture after hot rolling were almost completely wiped out by further cold rolling and annealing following a conventional processing route. It was also shown that further laser cutting altered the texture near the cut edge and caused deterioration in the magnetic characteristics of commercially produced electrical steel. 


\section{Acknowledgments}

Kim Verbeken is a Postdoctoral Fellow with the Fund for Scientific Research - Flanders (Belgium) (F.W.O.-Vlaanderen). G. Pasquarella (LCD Lasercut AG, CH-5026 Densbüren, Switzerland) is acknowledged for providing the commercially produced non-oriented electrical steels.

\section{References}

[1] M.R. Barnett and L. Kestens: ISIJ Vol. 39 (1999), p. 923.

[2] H. Homma and W.B. Hutchinson: Acta Mat. Vol. 51 (2003), p. 3795.

[3] Y. Hayakawa and M. Kurosawa: Acta Mat. Vol. 50 (2002), p. 4527.

[4] D. Dorner, S. Zaefferer and D. Raabe: Acta Mat. Vol. 55 (2007), p. 2519.

[5] H. Yashiki and A. Okamoto: IEEE T. Magn. Vol. 23 (1987) p. 3086.

[6] H. Huneus, K. Gunther, T. Kochmann, V. Plutniok and A. Schoppa: J. Mater. Eng. Perform. Vol. 2 (1993) p. 199.

[7] R. Kawalla, J. Schneider and A. Hensel: Proc. Workshop Metallurgy and Magnetism, TUBAF-Institut für Metallformung (2004).

[8] M. Kohno, M. Muraki, M. Kawano and M. Komatsubara: Mater. Sci. Forum Vol. 408-4 (2002) p. 785.

[9] J.T. Park, J.A. Szpunar and S.Y. Cha: ISIJ Int. Vol. 43 (2003) p. 1611.

[10] O. Fischer and J. Schneider: J. Magn. Magn. Mater. Vol. 254 (2003) p. 302.

[11] R. Kawalla and J. Schneider: Proc. 16th Intern. Conference SMM, Verlag Stahleisen GmbH Düsseldorf (2004).

[12] D. Vanderschueren, L. Kestens, P. Van Houtte, E. Aernoudt, J. Dilewijns, U. Meers: Textures And Microstructures Vol. 14 (1991) p.921.

[13] K. Verbeken, J. Schneider, J. Verstraete, H. Hermann and Y. Houbaert: IEEE T. Magn. Vol. 44 (2008) p. 3820.

[14] E. Gomes, J. Schneider, K. Verbeken, H. Hermann and Y. Houbaert: Mat. Sc. Forum (2009) accepted for publication.

[15] L. Kestens, Y. Houbaert, L. Delannay, P. Van Houtte, M.R. Barnett and J.J. Jonas: in: Proc. ICOTOM-12, edited by J.A. Szpunar, NRC Research Press, Montreal (1999) p. 910.

[16] K. Verbeken, L. Kestens and JJ. Jonas: Scr. Mater. Vol. 48 (2003) p. 1457.

[17] L. Kestens, Ph.D. dissertation, KU Leuven, 1994.

[18] E. Gomes, J. Schneider, K. Verbeken, J. Barros and Y. Houbaert: IEEE T. Magn. accepted for publication.

[19] J. Barros, J. Schneider, K. Verbeken and Y. Houbaert: J. Magn. Magn. Mater. Vol. 320 (2008) p. 2490.

[20] E. Gomes, J. Schneider, K. Verbeken, G. Pasquarella and Y. Houbaert: IEEE T. Magn. accepted for publication.

[21] P. Gobernado and L. Kestens, unpublished work. 